\title{
Expression of Class I and II Major Histocompatibility Complex (MHC) Antigens in the Developing CNS
}

\author{
A. Grabowska and L.A. Lampson \\ Center for Neurologic Diseases, Department of Neurology, Brigham and Women's Hospital and Harvard \\ Medical School, Boston MA 02115, USA
}

The clinical potential of neural transplantation depends upon the feasibility of allogeneic or xenogeneic transplants, particularly of fetal tissue. This, in turn, is influenced by donor cell expression of MHC antigens. MHC expression in the developing CNS in situ had not been defined. Here, a panel of antibodies was used to define $\mathrm{MHC}$ expression in the developing rat embryo.

\section{METHODS}

Rat embryos from gestation day 8 were examined by immunostaining. Multiple antibodies to $\mathrm{MHC}$ class I or b2-microglobulin (b2-m, the invariant chain of class I molecules) and class II antigens were used. They included monoclonals to class I and II, and rabbit antisera to b2-m. Cryoprotected frozen tissue (optimizing antigenicity), and paraffin-embedded tissue (optimizing morphology) were examined. Immunostaining conditions optimizing detection of cell surface or internal antigen were both used. Positive controls included brains that had been injected with cells of known MHC expression ("model tissue"). Monoclonals to leukocyte subsets were used in double-labeling studies, to further identify MHC+ cells.

\section{RESULTS}

MHC class I and II were detected in the surrounding maternal tissue from the earliest day examined (day 8), and in the developing embryo from day 13. MHC expression appeared first in liver and thymus, and in the connective tissue of the head. The earliest expression was seen before the appearance of peripheral $\mathrm{T}$ cells. With time, staining became more widespread. Outside lymphoid or hematopoietic tissues, a similar pattern of stain was always seen: The strongest stain was seen in individual cells in the connective tissue components of the developing organs. Overall staining of parenchymal cells was not seen. Developing brain and spinal cord were similar to other tissues: MHC class I and II were detected in individual cells in the associated connective tissues, but were not detected in developing neural cells.

Based on their distribution, morphologies, and staining patterns with anti-leukocyte antibodies, the class I+ and class II+ cells were identified as different subpopulations of mononuclear phagocytes.

\section{DISCUSSION}

In developing brain and spinal cord, MHC class I and II were detected in associated connective tissues, but not in neural cells. This is consistent with other studies in which strong MHC expression was not detected in adult, developing, or regenerating neural cells in situ /1/. Taken together, these findings argue against a non-immunological role for MHC proteins in neural development, regeneration or homeostasis. This suggests that measures designed to prevent MHC upregulation, as has been seen following surgical trauma or in vitro culture, need not inhibit growth or function of neural cells.

It is often suggested that lack of detectable MHC expression is a special property of neural cells. Our findings support an alternative view, 
that MHC expression is under regulatory control in all tissues. Yet another example is seen in normal adult skeletal muscle, where strong MHC expression is seen in endothelial cells, but is not detectable in muscle fibers themselves $/ 2 /$.

In the rat embryos, the MHC class $\mathrm{I}+$ and II + cells outside the lymphoid or hematopoietic tissues were identified as subpopulations of mononuclear phagocytes. A well-established function for $\mathrm{MHC}+$ mononuclear phagocytes in the adult is to serve as antigen-presenting cells. Under different conditions, stimulation or tolerance may result. The mechanisms for establishment and maintenance of tolerance to extra- thymic antigens, and of maternal/fetal tolerance, are still being defined. Their early appearance leads us to suggest that the MHC+ cells we have defined may play a role in both contexts. Studies designed to test this hypothesis will help to reveal the cells' potential function in transplanted neural tissue as well.

\section{REFERENCES}

1. Lampson LA et al. What function is served by MHC upregulation in neural cells? This volume (11.P1).

2. Lampson LA et al. Ann Neurol 1990; 28: 365-372. 

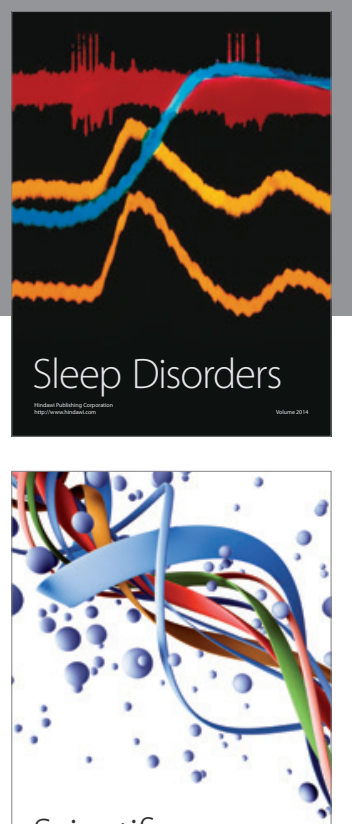

Scientifica
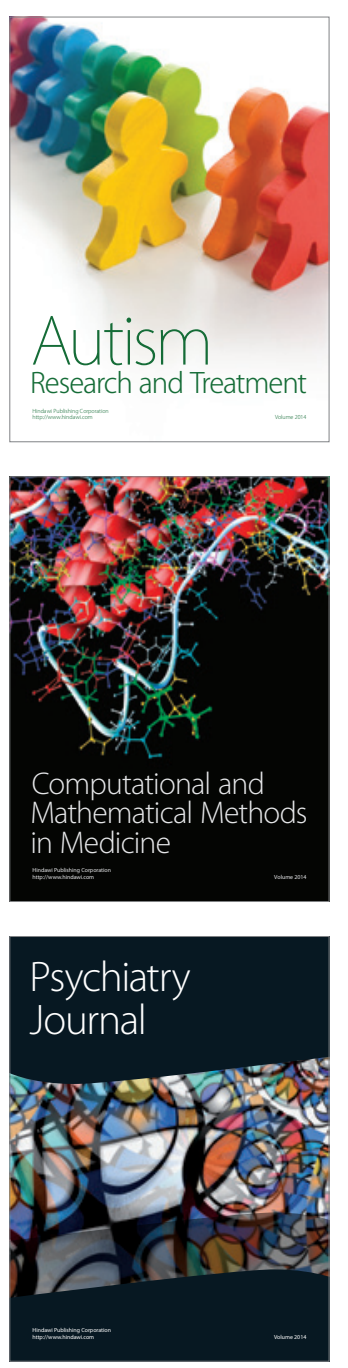
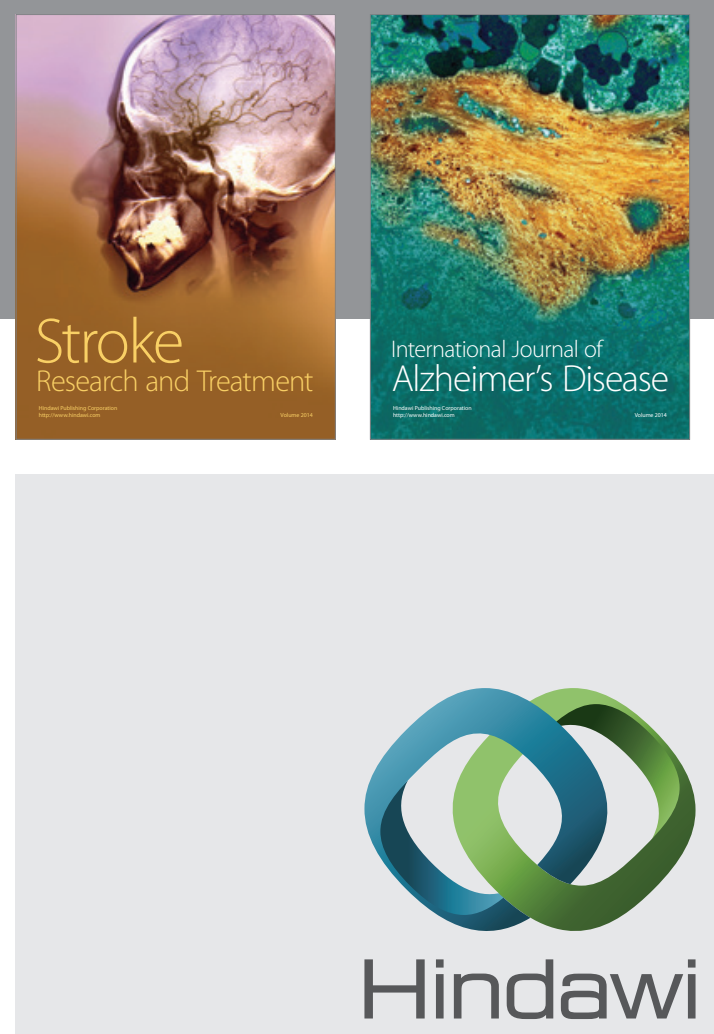

Submit your manuscripts at

http://www.hindawi.com
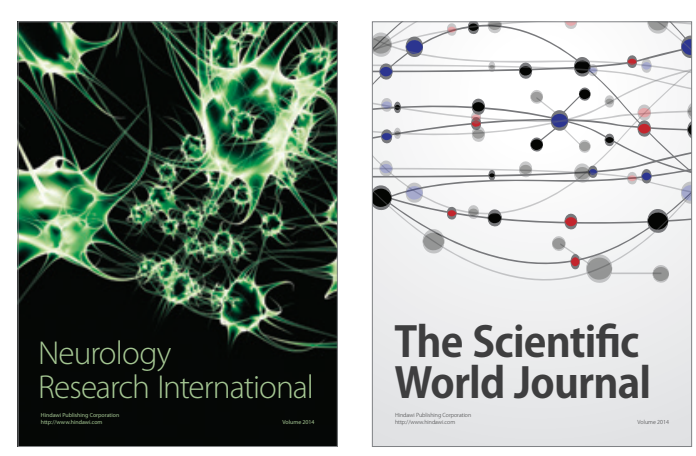

The Scientific World Journal

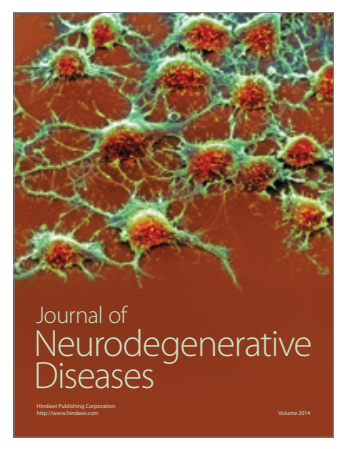

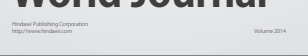

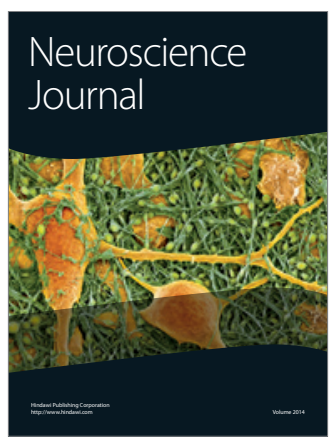

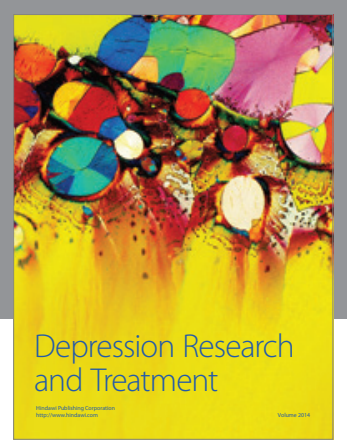
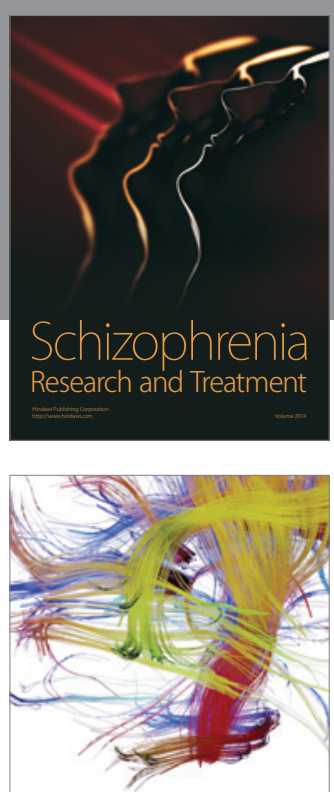

Brain Science

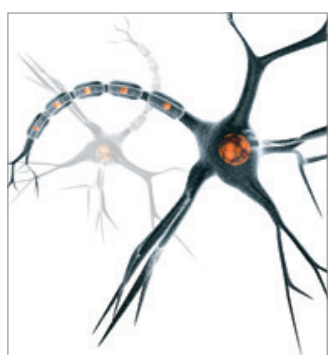

Neural Plasticity
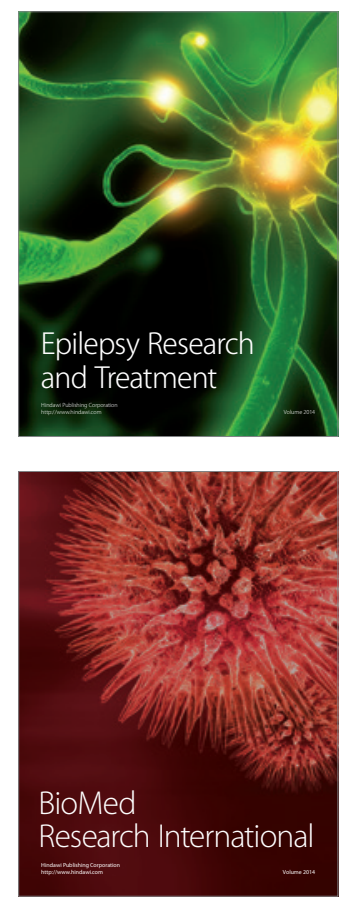

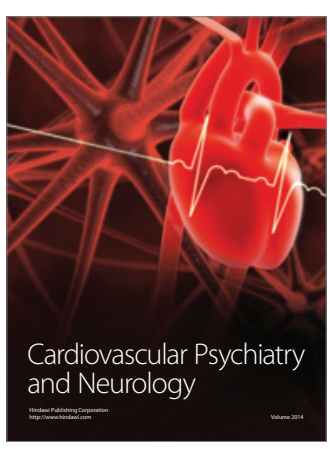

Parkinson's

Disease
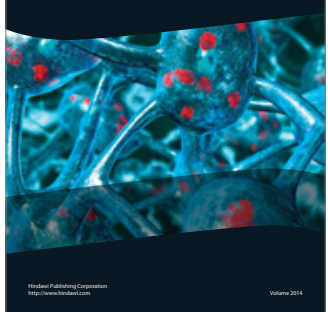